\section{Validación del auto-reporte de la localización de residuos sólidos con análisis de Procusto en el contexto de una iniciativa comunitaria participativa}

\author{
Validation of reported location of solid waste using \\ Procrustes analysis in the context of a participatory \\ community initiative
}

\author{
Validação do auto-relatório na localização \\ de resíduos sólidos com análise de Procusto \\ no contexto de uma iniciativa comunitária \\ participativa
}

Lilian E. Pacheco-Magaña 1

Álvaro J. Idrovo 2

Luz Arenas-Monreal 1

Marlene Cortez-Lugo 3

Luisa M. Sánchez-Zamorano ${ }^{3}$

\footnotetext{
1 Centro de Investigación en Sistemas de Salud, Instituto Nacional de Salud Pública, Cuernavaca, México.

2 Fundación Cardiovascular de Colombia, Floridablanca Colombia.

${ }^{3}$ Centro de Investigación en Salud Poblacional, Instituto Nacional de Salud Pública, Cuernavaca, México.

Correspondencia A. J. Idrovo Fundación Cardiovascular de Colombia.

Calle 155A № 23-58, Floridablanca / Santander, Colombia. alvaroidrovo@fcv.org
}

\begin{abstract}
This study is part of a community initiative for integrated solid waste management based on "popular epidemiology" and participatory action research. The study proposes a method for validation of spatial data ( $X$ and $Y$ data) on solid waste reported by community members. Location of sites was conducted by 76 residents of the La Nopalera community (Yautepec, Morelos, Mexico) and compared with reports by three environmental health experts. Agreement was explored using Procrustes analysis. Experts identified 23 sites with abundant solid waste, while community members only identified 22 sites. There were minimal differences in location, scale, and positioning of reported waste. Procrustes statistics showed 95\% agreement between community and expert reports. The findings suggest that community knowledge can be a valid source of information in epidemiological studies, and that measurement error can be quantified. The simple methodology described here can be used in further "popular epidemiology" studies or similar approaches.
\end{abstract}

Geographic Locations; Self Report; Validation Studies

\section{Resumen}

Este estudio forma parte de una iniciativa comunitaria de manejo integral de residuos, basada en "epidemiología popular" y la investigación acción participativa. En este estudio se propone un método de validación de datos espaciales (ordenadas y abscisas) de residuos sólidos autoreportados por la comunidad. La ubicación de los sitios fue realizada por 76 residentes de la localidad La Nopalera (Yautepec, Morelos, México), y comparada con los reportados por tres expertos en salud ambiental. La concordancia fue explorada con análisis de Procusto. Los expertos identificaron 23 sitios de concentración de residuos sólidos, mientras que la comunidad ubicó solo 22 sitios. Hubo mínimas diferencias en la localización, escala y orientación. El estadístico de Procusto mostró 95\% de concordancia entre legos y expertos. Los hallazgos sugieren que el saber popular es una fuente de información válida en estudios epidemiológicos, y que su error de medición puede ser cuantificado. La sencilla metodología descrita podrá ser usada en futuros estudios de "epidemiología popular" o aproximaciones similares.

Ubicaciones Geográficas; Autoinforme; Estudios de Validación 


\section{Introducción}

El auto-reporte es una fuente de información que desde el enfoque epidemiológico convencional suele ser visto como fuente de error de medición 1,2. Esto conlleva que los estudios con autoreporte suelan considerarse como poco válidos, y requerir mayores muestras para obtener un estimador de asociación confiable. Sin embargo, el auto-reporte ha sido útil en varios contextos 3,4, y es considerado un resultado de importancia en estudios de epidemiología social. La exactitud del auto-reporte de atributos personales es la que suele ser evaluada, mientras las variables espaciales no han recibido atención. Los estudios se limitan a evaluar si es o no correcta una localización anatómica ${ }^{5}$, sin considerar las dos o tres dimensiones que incorpora un espacio. Esto puede ser consecuencia del limitado desarrollo de los conceptos de espacio y lugar en epidemiología 6,7,8.

Los residuos sólidos fueron un problema identificado por la comunidad en un diagnóstico participativo ecosistémico 9,10, realizado entre 2007 y 2008 en La Nopalera (Yautepec, Morelos, México), por los habitantes y un equipo del Instituto Nacional de Salud Pública de México. Posteriormente, en 2009 se realizó una intervención comunitaria para manejar integralmente los residuos sólidos ${ }^{11}$. El presente estudio muestra los resultados de la validación de datos de localización espacial de acumulación de residuos.

La investigación-acción-participativa y la "epidemiología popular" brindaron sustento a este estudio 9,12. En este abordaje se acepta el conocimiento de legos sobre efectos de contaminantes en la salud; luego sus experiencias pueden ser corroboradas por expertos externos a la comunidad, quienes colaboran en las acciones que buscan tener un diagnóstico de la situación y en las intervenciones para mitigar el impacto de los contaminantes 13. Como puede apreciarse, la evaluación de la exactitud del reporte de ubicación espacial de lugares resulta ser de interés en el contexto de la "epidemiología popular".

\section{Métodos}

Se validaron los datos espaciales reportados por 76 miembros legos de la comunidad, en comparación con los reportados por tres expertos en salud ambiental. En esta localidad viven cerca de 600 habitantes, la mayoría dedicados a los cultivos de sorgo, maíz y agave, y la explotación de palma y madera ${ }^{14}$. El área geográfica habitada es aproximadamente de $0,4848 \mathrm{~km}^{2}$. A legos y exper- tos se les entregaron esquemas de la localidad, dividida en seis zonas denominadas con las primeras letras del alfabeto (Figura 1), con dimensiones de $43.18 \times 27.94 \mathrm{~cm}$, equivalente a una escala de 1:11 metros. Se dio la instrucción de que ubicaran lugares con residuos sólidos en abundancia; estos puntos críticos fueron considerados como los más adecuados para evaluar cambios asociados a una intervención comunitaria que se realizó posteriormente. El recorrido por la localidad fue realizado independientemente por ambos grupos, con una diferencia de 7-10 días, y en ambos casos se buscó un consenso interno que llevó a la identificación de los puntos.

Se usó el análisis de Procusto para comparar la información brindada por legos y expertos. Una explicación formal de los fundamentos del método se encuentra en textos especializados 15 . De manera sencilla, la comparación entre los dos esquemas se fundamenta en la evaluación de las diferencias en localización, escala y orientación 16, lo cual puede ser expresado como: Mapa de legos $=$ transformación

(mapa de expertos) + residuos donde la transformación busca minimizar al máximo posible los residuos, e incluye tres elementos: la translación, la escala y la rotación. En este estudio, la translación hace referencia a las diferencias que hay en la localización de los puntos reportados entre el esquema de legos y el de expertos, tanto en las abscisas como de las ordenadas. La evaluación de la escala incluye la identificación de un factor de multiplicación que permite igualarlos, y la rotación al desplazamiento de todos los puntos por un ángulo constante, manteniendo la distancia de cada punto del centro sin cambios. Esto está resumido en la Figura 2. Los análisis fueron realizados con el programa Stata 11 (Stata Corp., College Station, Estados Unidos).

\section{Resultados}

Los expertos identificaron 23 puntos y los legos 22 puntos. El punto e5 fue identificado exclusivamente por los expertos. En la Figura 3 se pueden apreciar esquemas realizados por legos y expertos para los puntos reportados. El promedio de valores en las abscisas entre expertos fue de 11.7 (desviación estándar 4.78), mientras que entre legos fue de 12.21 (desviación estándar 4.98); en ambos casos las abscisas estuvieron entre los valores 4.3 y 20.4. En el caso de los valores de las ordenadas, el promedio entre los expertos fue de 8.82 (desviación estándar 3.09, mínimo 4.2 y máximo 15) y de legos de 8.93 (desviación estándar 3.24, mínimo 4.2 y máximo 15.7). 


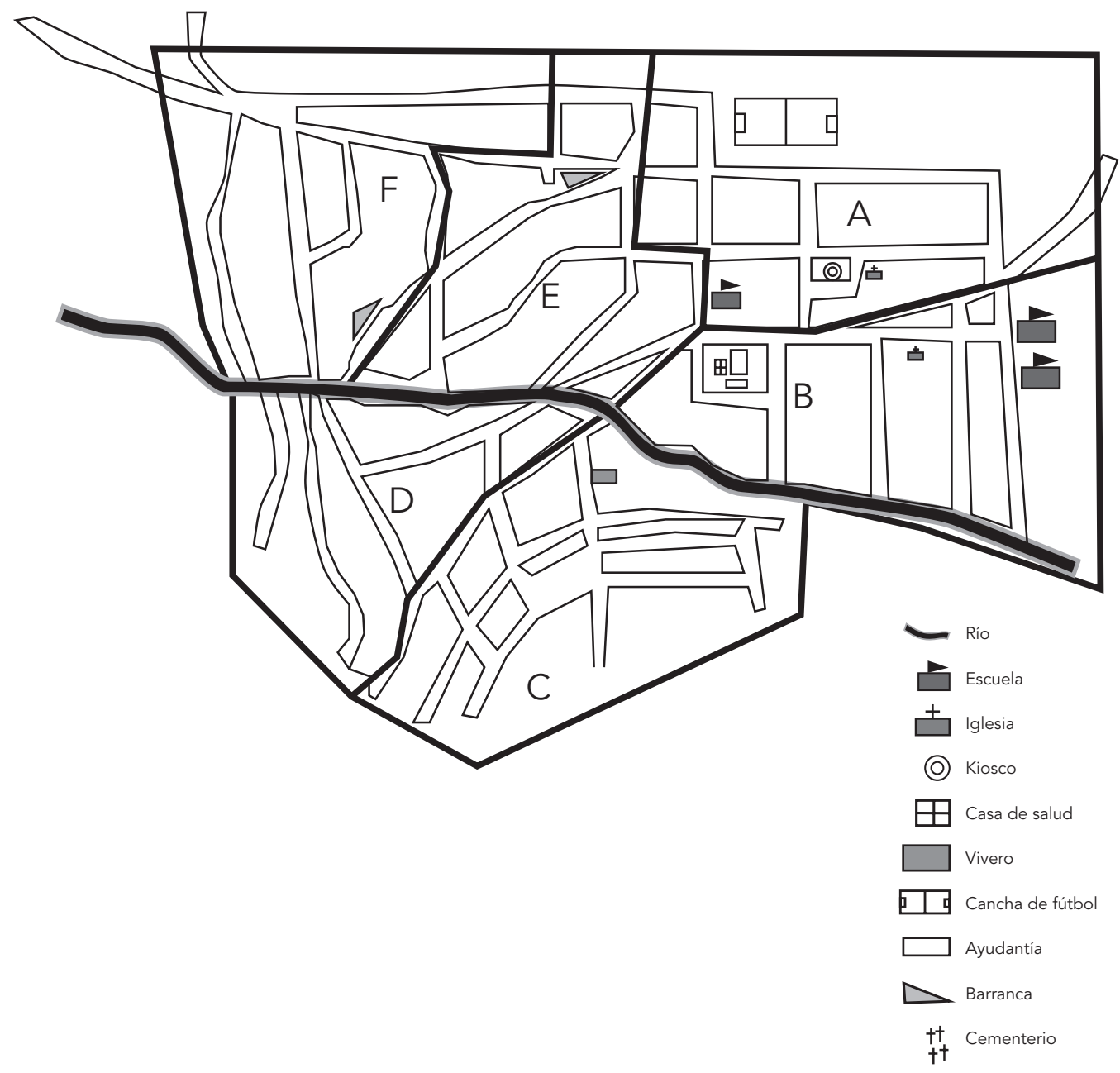

La translación fue mínima, siendo la diferencia de coordenadas entre legos y expertos de 0.047 en las abscisas y de 0.834 en las ordenadas. El factor de dilación (escala) fue de 0.9339, muy cercano a la igualdad en escala equivalente al valor uno. La orientación tampoco varió mucho, pues el giro entre las abscisas fue de 0.999, y en las ordenadas de 0.031 . El análisis estadístico global de Procusto, equivalente al complemento de la $r^{2}$ de los análisis de regresión lineal (1- $\left.r^{2}\right)$, dio un resultado de 0.050. Este mismo análisis estadístico para la abscisa y ordenada del experto, de manera independiente, tuvieron valores de 0.049 y 0.053, respectivamente (Figura 3).

\section{Discusión}

El principal resultado fue que ambos esquemas son parecidos; existió concordancia en $94.95 \%$ de los lugares con concentración de residuos sólidos. Esto implica que es posible predecir el conocimiento de expertos con los resultados de los legos. Los hallazgos de este estudio apoyan la idea que el auto-reporte de la comunidad sobre la localización de residuos sólidos es una forma válida de obtener información espacial. Las diferencias entre legos y expertos no resultó ser importante, como para definir los lugares prioritarios de evaluación de la intervención. No 


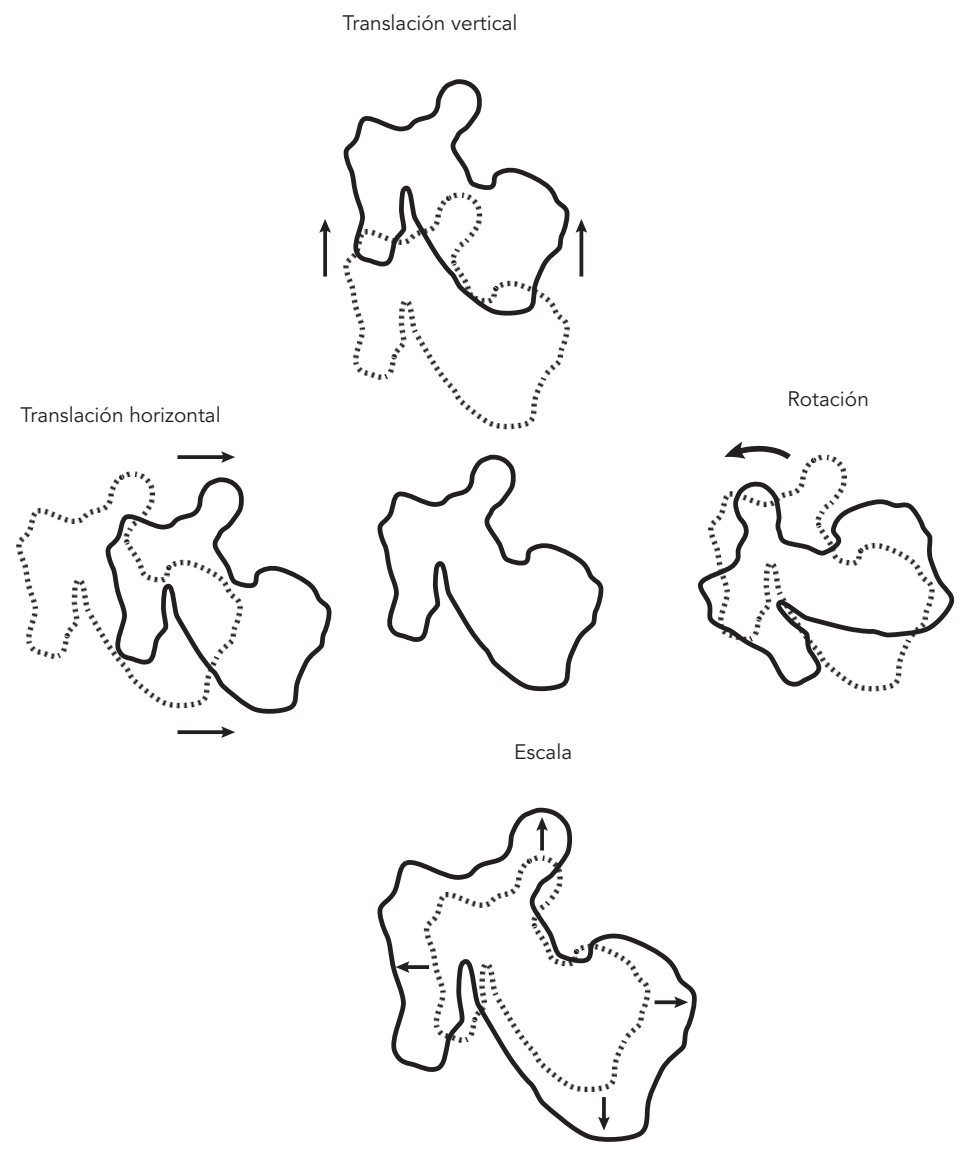

conocemos estudios similares con los cuales comparar estos hallazgos, por lo que este trabajo resulta ser el primero que realiza este tipo de evaluación. Sin embargo, los análisis aquí usados pueden ser complementados con técnicas etnográficas de mapeo espacial 17 .

La diferencia entre expertos y legos pudo ser debida a las diferencias en tiempo entre los dos recorridos, o que dicho punto no representó un problema de residuos sólidos para la comunidad por la cantidad de basura encontrada. Sin embargo, es notorio que 22/23 (95.65\%) de los puntos fueron ubicados por legos y expertos, con un ajuste casi perfecto según el análisis estadístico de Procusto. Es importante recordar que los esquemas originales eran relativamente pequeños en escala, por lo que es posible que con el uso de esquemas más grandes, que permitan un mejor detalle, se disminuya el ajuste. No obstante, los hallazgos del estudio sugieren que esto se reflejaría principalmente en el factor de dilación.

Este estudio comparte elementos semejantes a estudios de epidemiología popular, donde la comunidad identifica los problemas y propuestas de solución, manteniendo diálogo constante entre el equipo de investigación y la población 18 . Dentro de la epidemiología popular, los conocimientos locales cobran relevancia y deben ser tomados en cuenta. En conclusión, el autoreporte de lugares es una fuente confiable de información que puede ser usada en futuros estudios epidemiológicos; resulta especialmente útil cuando se usan aproximaciones como: la "epidemiología popular", "epidemiología alternativa”, "epidemiología feminista”, epidemiología crítica y la epidemiología con enfoque de género, que aceptan como válidas la información brindada por los propios sujetos de estudio. Esta 


\section{Figura 3}

Esquemas generados con los puntos críticos de concentración de residuos sólidos.
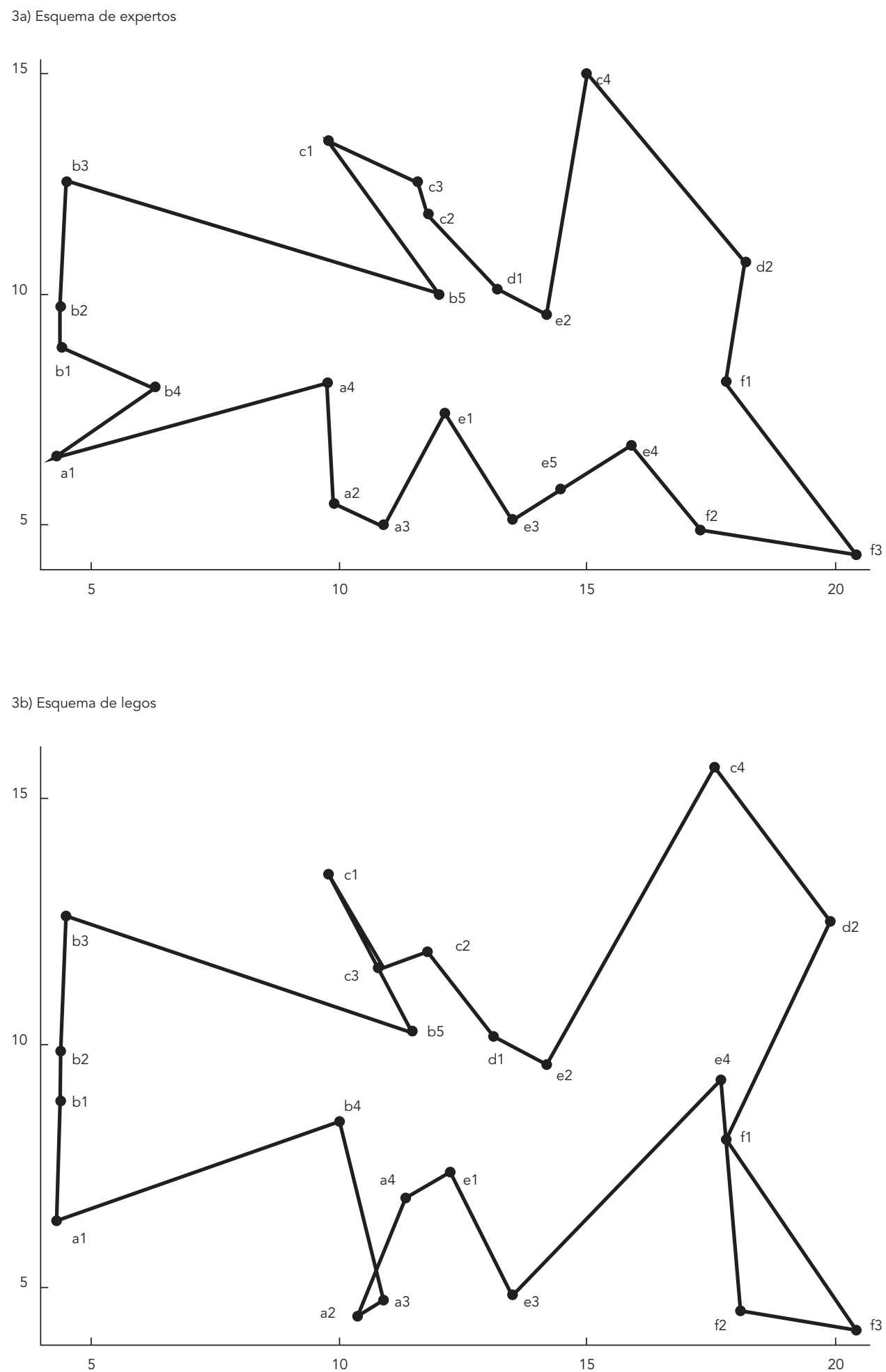
experiencia muestra que el auto-reporte puede ser una herramienta útil para los planes de gobierno municipal, estatal y nacional para enfrentar el manejo de residuos sólidos. Este estudio contribuyó a que la población gestionará recursos para la construcción de un centro de acopio de residuos sólidos.

\section{Resumo}

O estudo é parte de uma iniciativa comunitária de gerenciamento integral de resíduos sólidos, baseada na "epidemiologia popular" e em uma pesquisa de ação participativa. Aqui está sendo proposto um método de validação de dados espaciais (ordenadas e abscissas) de resíduos sólidos auto-relatados pela comunidade. A localização dos pontos foi realizada por 76 moradores da localidade de La Nopalera (Yautepec, Morelos, Méxi co), e comparada com os relatórios de três especialistas em saúde ambiental. A concordância foi explorada com análise de Procusto. Os especialistas identificaram 23 pontos de concentração de resíduos sólidos, ao passo que a comunidade localizou apenas 22 . Foram verificadas diferenças mínimas na localização, escala e orientação A estatística de Procusto mostrou 95\% de concordância entre leigos e especialistas. Os resultados sugerem que o conhecimento popular constitui uma fonte de informação válida em estudos epidemiológicos, e que seus erros de medição podem ser quantificados. A metodologia simples descrita poderá ser usada em futuros estudos de "epidemiologia popular" ou aproximações similares.

Localizações Geográficas; Auto-Relato; Estudos de Validação

\section{Colaboradores}

L. E. Pacheco-Magaña participó en el diseño, análisis e interpretación de los datos, redacción del artículo y aprobación de la versión final. A. J. Idrovo participó en el diseño, análisis e interpretación de los datos, redacción del artículo y aprobación de la versión final. L. ArenasMonreal participó en el diseño e interpretación de los datos, redacción del artículo y aprobación de la versión final. M. Cortez-Lugo participó en el diseño e interpretación de los datos, redacción del artículo y aprobación de la versión final. L. M. Sánchez-Zamorano participó en el diseño e interpretación de los datos, redacción del artículo y aprobación de la versión final.

\section{Agradecimientos}

Los autores agradecen la participación de los habitantes de La Nopalera, al maestro Ing. Luis Camilo Blanco de la Universidad Industrial de Santander (UIS) y al Lead Graphic Designer Ernesto Pacheco por su apoyo con las ilustraciones vectoriales. Este trabajo es parte de las prácticas comunitarias que realizan los estudiantes de salud pública del Instituto Nacional de Salud Pública/ Escuela de Salud Pública de México. 


\section{Referencias}

1. Wacholder S. When measurement errors correlate with truth: surprising effects of nondifferential misclassification. Epidemiology 1995; 6:157-61.

2. Wing S. Limits of epidemiology. Med Global Survival 1994; 1:74-86.

3. Idler E, Kasl S. Self-ratings of health: do they also predict changes in functional ability? J Gerontol B Psychol Sci Soc Sci 1995; 50:S344-53.

4. Miilunpalo S, Vuori I, Oja P, Pasanen M, Urponen H. Self-rated health status as a health measure: The predictive value of self-reported health status on the use of physician services and on mortality in the working-age population. J Clin Epidemiol 1997; 50:517-28.

5. Chesbrough RM, Burkhard TK, Balsara ZN, Goff 2nd WB, Davis DJ. Self-localization in US of appendicitis: an addition to graded compression. Radiology 1993; 187:349-51.

6. Cummins S, Curtis S, Diez-Roux AV, Macintyre S. Understanding and representing "place" in health research: a relational approach. Soc Sci Med 2007; 65:1825-38.

7. Czeresnia D, Ribeiro AM. O conceito de espaço em epidemiologia: uma interpretação histórica e epistemológica. Cad Saúde Pública 2000; 16:595-617.

8. Frohlich KL, Corin E, Potvin L. A theoretical proposal for the relationship between context and disease. Sociol Health Illn 2001; 23:776-97.

9. Forget G, Lebel J. An ecosystem approach to human health. Int J Occup Environ Health 2001; 7(2 Suppl):S3-38.

10. Arenas-Monreal L, Cortez-Lugo M, Parada-Toro I. Community-based participatory research and the Escuela de Salud Pública in Mexico. Public Health Rep 2011; 126:436-40.
11. Pacheco-Magaña LE. Manejo integral de los residuos sólidos para contribuir al control de enfermedades asociadas a estos, en la población de la localidad La Nopalera, Yautepec, Morelos bajo el enfoque de monitoreo participativo. Cuernavaca: Instituto Nacional de Salud Pública; 2009.

12. Brown P. Popular epidemiology and toxic waste contamination: lay and professional ways of knowing. J Health Soc Behav 1992; 33:267-81.

13. Leung MW, Yen IH, Minkler M. Community based participatory research: a promising approach for increasing epidemiology's relevance in the 21 st century. Int J Epidemiol 2004; 33:499-506.

14. Cervín-García V, Esparza-Lagunas L, Espinosa-Cárdenas F, Pacheco-Magaña LE, Wilches JL, Parada I, et al. Experiencia de diagnóstico integral participativo y con enfoque ecosistémico en Morelos. In: Arenas L, Sosa N, Corrales A, editores. Experiencias de acercamiento comunitario y participación de la población en salud. Cuernavaca: Instituto Nacional de Desarrollo Social/Secretaría de Desarrollo Social/Instituto Nacional de Salud Pública; 2008. p. 50-65.

15. Dryden IL, Mardia KV. Statistical shape analysis. New York: J.W. Wiley; 1998.

16. Cromley EK. Mapping spatial data. In: Schensul, J, LeCompte M, Trotter R, Cromley E, Singer M, editors. Mapping social networks, spatial data \& hidden populations. Walnut Creek: Altamira Press; 1999. p. 51-124.

17. Browne MW. On oblique Procrustes rotation. Psychometrika 1967; 32:125-32.

18. De Roux GI. Participación y congestión de la salud Educ Med Salud 1993; 27:50-60.

Recibido el 05/Ene/2012

Versión final presentada el 03/Ago/2012

Aprobado el 11/Sep/2012 